\title{
La escritura en el tratamiento psicopedagógico: producción simbólica y proceso identificatorio
}

A escrita no tratamento psicopedagógico: produção simbólica e processo de identificação

Writing at psychopedagogic treatment: symbolic production and recognition process

Julián Grunin*

\begin{abstract}
Resumen
Se presentan resultados de una tesis doctoral, subsidiada por el Conicet (Ministerio de Ciencia), y desarrollada en la Cátedra Psicopedagogía Clínica con sede en la Facultad de Psicología de la Universidad de Buenos Aires (UBA). En la misma se exploraron las formas actuales de abordaje clínico sobre las problemáticas de simbolización de púberes y adolescentes con dificultades de aprendizaje. El trabajo clínico con los jóvenes consultantes generó nuevos interrogantes teóricos que exigieron ampliar el campo de las investigaciones para reflexionar sobre las estrategias específicas de intervención en la clínica contemporánea, así como de transferencia al campo educativo. En el presente artículo se desarrollarán, por ende, resultados investigativos sobre las características de su producción simbólica escritural (narrativa y figurativa) desplegada durante dos años de su tratamiento psicopedagógico grupal.
\end{abstract}

Palabras clave: Escritura. Simbolización. Identidad.

\section{Resumo}

Apresentam-se resultados de uma tese de doutorado subsidiada pelo Conicet (Ministério de Ciência), desenvolvido na disciplina Psicopedagogia Clínica, da Faculdade de Psicologia da Universidade de Buenos Aires (UBA). Foram exploradas formas atuais de abordagem clínica sobre questóes de simbolização de púberes e adolescentes com dificuldades de aprendizagem. O trabalho clínico com pacientes jovens gerou novas questōes teóricas que exigiram ampliar o escopo da pesquisa para refletir sobre estratégias de intervenção específicas na clínica contemporânea e permitiram transferi-las para o campo educacional. Neste artigo, vamos desenvolver, portanto, resultados de pesquisas sobre as características de sua produção simbólica escrita

Doctor en Psicología, Universidad de Buenos Aires (UBA), Argentina. Consejo Nacional de Investigaciones Científicas y Técnicas (Conicet). Ministerio de Ciencia, Tecnología e Innovación Productiva de la Nación. Cátedra Psicopedagogía Clínica, Facultad de Psicología de la Universidad de Buenos Aires (UBA). Fuente de financiamiento: Conicet. Se reconoce el apoyo técnico para la traducción del resumen en español al resumo en portugués de la Lic. Aline de Oliveira Perotto. 
(narrativa e figurativa), desenvolvidas durante dois anos, no grupo de tratamento psicopedagógico.

Palavras-chave: Escrita. Simbolização. Identidade.

\begin{abstract}
We present results of a doctoral thesis, subsidized by the CONICET (Ministry of Science), and developed at the Faculty of Psychology at the University of Buenos Aires (UBA). Via these same ways were explored current clinical approaches on issues of symbolization of pubescent and adolescents with learning disabilities. Clinical work with young patients generated new theoretical questions that demanded broadening the scope of research to reflect on specific intervention strategies in contemporary clinical and transfer to the educational field. In this article we develop, thereupon research results on the characteristics of their writing symbolic production (narrative and figurative) deployed for two years psycho-pedagogic treatment group.
\end{abstract}

Keywords: Writing. Symbolization. Identity.

\title{
1. Introducción y objetivos de la investigación
}

Se presentan los resultados de una tesis doctoral (Grunin, 2013) ${ }^{1}$ que tuvo por objetivo caracterizar las modalidades de simbolización involucradas en las producciones escriturales de púberes y adolescentes con problemas de aprendizaje, focalizando - en particular - en el abordaje de sus alcances identitarios en cada caso clínico. Para ello, se analizó la actividad escritural que los jóvenes consultantes desplegaban en sus cuadernos de trabajo a lo largo de dos años de su tratamiento psicopedagógico grupal.

El recorte investigativo propuesto recibió el subsidio del Conicet (Consejo Nacional de Investigaciones Científicas y Técnicas del Ministerio de Ciencia), a través de dos becas doctorales (2008-2013) y una postdoctoral (2013-2015), que contaron con la dirección de la Dra. Silvia Schlemenson y la co-dirección de la Dra. Patricia Álvarez (ambas profesoras de la Cátedra Psicopedagogía Clínica de la Facultad de Psicología de la Universidad de Buenos Aires, Argentina).

Los desarrollos de la tesis fueron producto del trabajo conjunto realizado en el marco del Programa de Investigación de la Cátedra Psicopedagogía

\footnotetext{
Tesis doctoral por la Facultad de Psicología de la Universidad de Buenos Aires (UBA): "Escritura y proceso identificatorio en la clínica de púberes y adolescentes con problemas de aprendizaje”. Directora de tesis: Dra. Silvia Schlemenson.
} 
Clínica, el cual se desarrolla (con vigencia desde el año 1988) en el Servicio de Asistencia Psicopedagógica dependiente de la Secretaría de Extensión Universitaria de la Facultad de Psicología de la Universidad de Buenos Aires (UBA). En dicho Servicio, se asiste a niños y adolescentes que presentan dificultades en sus procesos de aprendizaje, quienes son derivados por las distintas escuelas públicas de la Ciudad Autónoma de Buenos Aires para su diagnóstico y tratamiento psicopedagógico.

Actualmente, la Cátedra Psicopedagogía Clínica desarrolla (bajo la dirección de la Dra. Silvia Schlemenson) distintos proyectos de investigación - subsidiados por UBACyT (2011-2014)² y la ANPCyT (2010-2013) $)^{3}$ - que intentan generar estrategias de complejización en sus modalidades de producción simbólica (escritural, discursiva, gráfica y lectora), tal que favorezcan - durante su tratamiento - la remisión de las restricciones en sus procesos de simbolización que obstaculizan el despliegue de sus procesos imaginativos, reflexivos y de autonomía de pensamiento; necesarios éstos para todo aprendizaje e intercambio dúctil con los objetos sociales y de conocimiento (Schlemenson, 2009).

En el presente artículo se desarrollará, en particular, la fundamentación teórica de las dimensiones de estudio construidas para abordar - durante el proceso investigativo - la escritura de los jóvenes ("escritura narrativa” y "figurativa de los márgenes"). Se partió del supuesto que las mismas comprometían modalidades singulares de elaboración de sentido vinculadas a la tramitación de sus procesos de construcción identitaria.

Desde una perspectiva psicoanalítica, el proceso identificatorio resulta característico de una etapa particular del desarrollo (como es la adolescencia) de intensas transformaciones - corporales, identitarias, sociales, entre otras - que exigen de nuevas construcciones de sentido para su tramitación psíquica (Gutton, 1993; Aulagnier, 1991).

Se decidió focalizar el análisis en las marcas que cada paciente producía en momentos de escribir en el cuaderno durante cada sesión de su tratamiento. Se realizó, por lo tanto, un análisis exploratorio de su producción escrita, permitiendo elaborar mediaciones conceptuales específicas entre sus modalidades de simbolización y los procesos de construcción identitaria expresados en sus aspectos narrativos (del centro del cuaderno) y figurales

\footnotetext{
Proyecto UBACyT (2011-2014): "Procesos imaginativos en la producción simbólica de niños y adolescentes con problemas de aprendizaje”. Subsidiado por la Secretaría de Ciencia y Técnica de la UBA. Directora: Dra. Silvia Schlemenson.

Proyecto PICT (2010-2013): “Tratamiento psicopedagógico en niños y adolescentes en situación de pobreza”. Subsidiado por la Agencia Nacional de Promoción Científica y Tecnológica del Ministerio de Ciencia, Tecnología e Innovación Productiva. Directora: Dra. Silvia Schlemenson.
} 
(de sus márgenes posibles) (Grunin \& Schlemenson, 2011a, 2011b).

El trabajo investigativo en la clínica psicopedagógica se emprende - para ello - en un eje interdisciplinario, aunque orientado a generar herramientas teóricas específicas para el abordaje de las problemáticas de simbolización, que permitan la construcción de estrategias clínicas de intervención para su resolución (Schlemenson, 2009).

\section{Método y objeto: un encuentro complejo}

El proceso de la investigación se inscribió en un diseño de trabajo cualitativo, para poder emprender un estudio exploratorio y longitudinal de desarrollo de casos clínicos (González Rey, 2006), orientado a caracterizar la incidencia de la escritura adolescente en los procesos de elaboración de sentidos subjetivos de alcance identitario.

Uno de los aspectos que caracterizan la producción del conocimiento en la investigación cualitativa es la atención al carácter singular de lo estudiado, que se expresa en la legitimidad que se le atribuye al estudio de casos como momento de la investigación científica (González Rey, 1999, p. 74).

Los resultados de la investigación se sustentaron en un proceso de análisis cualitativo, exploratorio y longitudinal del material clínico recolectado en 49 sesiones grupales que fueron registradas (por videofilmación) y desgrabadas en su totalidad.

El registro y la desgrabación de cada sesión, respondió -ante todoa la necesidad metodológica de construcción de categorías de análisis e indicadores que permitieron el abordaje de cada dimensión de estudio propuesta (Álvarez, 2010).

La propuesta metodológica se fundamentó en los principios de la epistemología cualitativa, que permitieron destacar el carácter constructivointerpretativo del proceso de producción de hipótesis (en este caso, sobre las modalidades de elaboración de sentidos identitarios) en el realce específico - no unidireccional - de los elementos indiciarios convergentes en el material clínico estudiado (escritura) (González Rey, 1999).

Se construyeron, asimismo, indicadores y ejes de análisis específicos que organizaron el abordaje de las dos dimensiones de estudio propuestas (escritura narrativa y figurativa) para dar cuenta de la heterogeneidad propia del objeto de estudio. Las categorías elaboradas para el análisis 
de la escritura adolescente, en su relación específica con los procesos de construcción identitaria, permitieron - en este sentido - generar mediaciones conceptuales entre los indicios realzados en el material clínico y las hipótesis construídas para su interpretación en la clínica.

Por tratarse de un diseño delimitado por el tipo de encuadre clínico, se partió de un criterio de selección muestral teórico, no probabilístico y de carácter intencional (Taylor \& Bogdan, 1992). El grupo seleccionado estuvo compuesto por tres jóvenes (de género femenino y masculino) de entre 11 y 14 años que asistieron semanalmente, durante dos años, al servicio asistencial que se desarrolla con sede en la Facultad de Psicología de la UBA.

El universo del que se seleccionó la muestra estuvo integrado por la población a la cual está dirigida el área asistencial pública, que la Cátedra Psicopedagogía Clínica coordina con sede en la UBA, compuesta por púberes y adolescentes que cursan en escuelas secundarias públicas de la Ciudad Autónoma de Buenos Aires, y que son derivados a los respectivos equipos psicopedagógicos a partir de la detección de problemas en sus procesos de aprendizaje escolar.

El propósito de la selección se justificó por la viabilidad del modelo teórico específico de la presente investigación. En esta línea, el criterio de selección resultó homogéneo en cuanto a las dificultades de aprendizaje que presentaban los pacientes jóvenes en el contexto escolar, así como en relación a las condiciones socioeconómicas desfavorables de los jóvenes y sus familias que nos consultan, y heterogéneo en cuanto a la complejidad y diversidad de los procesos psíquicos comprometidos en cada problemática singular. Por lo tanto, el muestreo aquí propuesto fue deliberado y basado en la significatividad (Cayssials, 2010) de los casos clínicos seleccionados.

La recolección de los datos estuvo compuesta por distintos tipos de fuentes: Por un lado, las distintas producciones escritas que los sujetos realizaban en sus propios cuadernos de tratamiento y que resultaron significativas según las dimensiones de análisis de las modalidades de simbolización comprometidas. Y por el otro, se seleccionaron producciones dialógicas recortadas de distintas situaciones de las sesiones de tratamiento videograbadas, y posteriormente desgrabadas para su análisis, en tanto permitieron el análisis de las asociaciones producidas sobre las marcas escriturales elaboradas en sus cuadernos.

En el caso de la producción escrita, el material de análisis se constituyó por las producciones narrativas (ficcionales $\mathrm{u}$ autorreferenciales) que 
cada joven desplegó en su cuaderno de trabajo como respuesta a las distintas intervenciones y consignas de trabajo que los terapeutas elaboran en cada sesión grupal.4 Se interroga aquí sobre los procesos psíquicos necesarios para que cada sujeto se apropie del código escrito, es decir de las significaciones socialmente compartidas, de modo que le permita el despliegue creativo de su subjetividad en la creación de sentidos propios, singulares, organizados de acuerdo a la legalidad de dicho código (Cantú, 2011).

Partiendo, a su vez, de uno de los hallazgos investigativos de la tesis (Grunin, 2013), se consideró también como material de análisis de la escritura, a aquellas marcas figurativas espontáneas que -en especial los adolescentes producían en los márgenes de sus cuadernos de trabajo durante las sesiones de su tratamiento, como expresiones distintivas y creativas de sus procesos imaginativos vinculados a los trabajos de construcción identitaria (marcas de autoría relacionadas a ensayos de firmas, re-creación del nombre propio y/o de pares, invención de emblemas identitarios, entre otras tantas).

La edad de los sujetos constituyó, también, un criterio de selección muestral, ya que se consideró que los procesos imaginativos y de construcción identitaria constituyen uno procesos psíquicos principales de la adolescencia, en un período vital del desarrollo que plantea exigencias inéditas de construcción de sentido, y de proyección de alternativas identitarias en la configuración de nuevas trayectorias de inclusión social y educativa.

Los casos clínicos seleccionados resultaron distintivos de las problemáticas de simbolización de la pubertad y la adolescencia. En esta línea, los jóvenes que asistieron al grupo seleccionado presentaban restricciones simbólicas que empobrecían su acceso a formas dúctiles de pensamiento, reflexión e imaginación. En su especificidad, sus modalidades de simbolización presentaron restricciones que oscilaban, con frecuencia, entre la prevalencia de inhibiciones simbólicas que empobrecían la autoría de sus producciones escriturales (así descriptivas, escuetas, o bien ausentes) o bien, por otro lado, la emergencia de aspectos de descarga en el cuaderno (tachaduras, borroneados, perforaciones de las hojas) que limitaban, tanto el despliegue de la propia actividad de pensamiento para la expresión subjetiva de sus principales conflictivas, como el intercambio con los otros semejantes.

Según la Dra. Alicia Cayssials (2010), la perspectiva cualitativa hace foco

La consigna de trabajo (individual, grupal y/o colectiva), realza - en cada sesión - las conflictivas que se despliegan en los intercambios iniciales de cada encuentro, para entonces ampliar las oportunidades elaborativas sobre las mismas a través de propuestas de escritura que se despliegan en el cuaderno. El tipo de respuesta a la consigna es singular. Puede ser escrita, aunque también gráfica; pudiendo - incluso - combinar diversos recursos figurativos al interior de una misma producción. 
en el proceso y la dinámica de las relaciones complejas con el objeto abordado. Dicha perspectiva destaca el interés en lo subjetivo, como eje sobre el que se organiza la producción interpretativa (más que el "descubrimiento") de conocimientos que -en esta línea- resultan no generalizables.

Enmarcada en la epistemología de la complejidad (Morin, 2000), la investigación se asoció - por lo tanto - a una metodología cualitativa por la cual la producción del método y las categorías de análisis se establecen, necesariamente, en el intercambio dialógico permanente con el material de estudio y los aportes del marco teórico en el cual arraiga la fundamentación de su análisis.

Para elaborar hipótesis clínicas sobre las problemáticas de simbolización en estudio, se profundizó - por lo tanto - en el análisis de las diferentes formas de elaboración de sentido subjetivo que los jóvenes presentaban en sus producciones escriturales. Se decidió, entonces, intervenir sobre la escritura narrativa y figural por considerar que resultan formas específicas de producción simbólica elegidas por los jóvenes para la expresión de sus conflictivas preponderantes en narrativas y otras marcas singulares de autoría y (como sus escrituras en los márgenes del cuaderno) que caracterizan los trabajos de ensayo sobre el propio registro identitario durante la adolescencia.

\section{Problemáticas clínicas}

El proceso investigativo se inscribió en la perspectiva teórica del psicoanálisis contemporáneo (Green, 2010; Aulagnier, 1994; Castoriadis, 1993a), desde la cual pudo fundamentarse el abordaje de los procesos psíquicos involucrados en las modalidades de simbolización de cada sujeto, y de sus restricciones patológicas cuando empobrecen su actividad representativa en el intercambio con los objetos sociales y de conocimiento.

Entre las problemáticas clínicas que asistimos en el Servicio de Psicopedagogía de la Facultad de Psicología UBA, se destacan modalidades restrictivas de simbolización en la producción simbólica (modos de escribir, leer, dibujar) de los púberes y adolescentes que nos consultan debido a sus dificultades en el aprendizaje escolar (Schlemenson \& Grunin, 2013).

Dichas modalidades suelen circunscribir el investimiento de los objetos secundarios a formas rígidas de intercambio con los mismos (en ocasiones sobreadaptadas), que tienden - incluso - a obturar la movilidad del afecto respecto al propio trabajo de pensamiento, derivando así en modalidades rígidas de elaboración de sentido sobre la propia experiencia, que - frecuentemente 
- se hallan desprovistas de índices subjetivos y libidinales. Por ejemplo, se destacan: dibujos a predominio de la copia, escritos de tipo descriptivos, asociaciones verbales remitidas a elementos factuales externos con escasa articulación en procesos imaginativos singulares, producciones lectoras a predominio de la repetición de la información literal del texto, entre otros indicios convergentes (Wald, 2010; Álvarez, 2010; Cantú, 2011).

Asimismo, cuando se distinguen modalidades fragmentarias de simbolización, estas suelen comprometer (restrictivamente) el acceso al proceso secundario, empobreciendo así las oportunidades de organización y transmisibilidad de sentidos propios a través de la escritura, el discurso, le lectura o la actividad proyectiva gráfica.

En esta línea, y en algunos casos clínicos, la dinámica del trabajo psíquico suele hallarse al servicio de tramitar la intensidad de cargas afectivas que no encuentran vías adecuadas de ligadura y procesamiento en representaciones. El fracaso del trabajo representativo puede, así, manifestarse a través de la tendencia a la descarga evacuativa en la acción directa (tachaduras en los dibujos, perforaciones del cuaderno, entre otros).

En este campo complejo de problemáticas clínicas, el investimiento de la producción simbólica tiende a fracasar en su función de mediación de la actividad fantasmática en sentidos compartidos hacia el exterior (Álvarez, 2010).

Por lo tanto, cuando el afecto resulta rígidamente escindido respecto al trabajo de representación, preponderan problemáticas de simbolización que fracturan el lazo con los objetos secundarios, derivando en: inhibiciones, irrupciones evacuativas de la actividad fantasmática en el pensamiento, somatizaciones y/o desinvestiduras masivas con efectos de vacio representacional, entre otras (Green, 1996, 2000, 2010).

En esta línea, se partió de la hipótesis que las modalidades de simbolización de los jóvenes consultantes, podían evaluarse -de forma exploratoria, cualitativa y longitudinal- en los distintos modos de expresión de su actividad escritural desplegada en los centros y márgenes de sus respectivos cuadernos de trabajo durante el tratamiento psicopedagógico (Schlemenson \& Grunin, 2011a, 2011b).

\section{Escritura, imaginación y proceso identificatorio}

Para Piera Aulagnier (1977, 1984, 1986), el proceso identificatorio da origen al advenimiento del yo como instancia enunciante. Entrada en escena 
de un tiempo historizado que expresa el acceso al registro simbólico de la temporalidad. Desde esta perspectiva, el trabajo de historización resalta el carácter indisociable del movimiento temporal respecto al movimiento libidinal de investiduras que activa el proceso mismo de simbolización. En esta línea, Aulagnier $(1977,1994)$ confiere al yo un potencial identificante. Plantea aquí al proyecto identificatorio como un trabajo continuo, por parte del yo, de invención de representaciones identitarias que condensan la investidura de un tiempo pasado, con la proyección (anhelo) de un tiempo por venir que anuda una expectativa sustitutiva de ganancia de placer propulsora de la actividad de simbolización:

El pasaje de un posicionamiento en que predomina el yo Ideal, posicionamiento fundamentalmente narcisista según el cual el yo se iguala al ideal, al del Ideal del yo, incluye la noción de proyecto. La distancia entre el yo actual y el ideal buscará ser zanjada a futuro. Futuro que se dibuja como proyecto identificatorio y como sede de ideales que habrán de funcionar como horizonte desiderativo para un yo en movimiento hacia lo porvenir" (Sternbach, 2006, p. 64).

Según Phillipe Gutton (1991, 1993), el trabajo de la adolescencia adquiere la especificidad de dar elaboración psíquica a la disrupción de la energía pulsional reactivada con intensidad durante el proceso puberal.

Se alude aquí a la noción de trabajo identificatorio para referir al trabajo de ensayo que orienta - entre movimientos de permanencia y cambio (Aulagnier, 1986) - los procesos de elaboración de alternativas sobre las formas instauradas de representar(se) y proyectar(se) temporalmente, durante un período de intensas transformaciones como es la adolescencia. Según Liliana Palazzini (2006):

La noción de trabajo es medular en la teoría psicoanalítica; contiene la idea de movimiento pulsional, de construcción representacional, de dinámica en juego, de creación, de elaboración. Lleva implícita la noción de fuerza en el interior del aparato [...] (p. 139).

La adolescencia involucra, en esta línea, transformaciones en los modos de circulación y tramitación pulsional, que movilizan exigencias inéditas de trabajo psíquico para simbolizar los cambios a nivel del cuerpo, el lugar del otro y el registro identificatorio:

La identidad, resultante del anudamiento de distintos registros, biológico, social y subjetivo, no es una esencia estable [...], sino 
un trabajo psíquico y social, que está siempre reformulándose, por el cual cada sujeto no cesa de construirse y de ser construido, poniendo en juego: herencia y creación, continuidad y ruptura (Frigerio, 2008, p. 61-62).

En síntesis, durante la adolescencia, los procesos de reorganización identitaria comprometen intensas transformaciones, que conmueven la continuidad temporal de las referencias identificatorias primarias (Rother, 2006; Lewkowicz, 1999), exigiendo de nuevas construcciones de sentido para la invención de nuevos emblemas y referencias de interés libidinal.

Se partió, entonces, del supuesto que el estudio de la escritura de los jóvenes consultantes permitía distinguir modalidades distintivas de elaboración de sentido, que involucran - a su vez-alternativas de tramitación (sobre elementos indiciarios particulares de sus problemáticas identitarias) que pudieron caracterizarse durante el tratamiento psicopedagógico grupal (Grunin,2013).

Según Sophie de Mijolla Mellor (1991, p. 84): "El proyecto identificatorio y la versión que el sujeto se forma de su propia historia son profundamente retocados y reorganizados. La escritura acompaña y trae su apoyo a esta reelaboración”. Para Phillipe Gutton (1991), la escritura posee - en la adolescencia - una función identificante. En la misma línea, Piera Aulagnier (1984) atribuye al yo una tarea de escritor que resalta, ante todo, su cualidad activa (historizante) en el esbozo singular, parcial y selectivo, de su propia historia:

Castoriadis destaca que el proceso identificatorio se produce entre dos polos: el primero es el de las significaciones imaginarias sociales, pero en el otro polo (la imaginación radical) éstas son mediatizadas por la propia historia del sujeto y son sometidas a la acción creadora de la imaginación (Franco, 2004, p. 314).

La escritura constituye, por tanto, una forma compleja de producción simbólica que expresa formas distintivas de la actividad psíquica de un sujeto (Schlemenson, 2009).

La propuesta de escritura, en el encuadre de la clínica psicopedagógica, intenta - en esta línea- ampliar las oportunidades de simbolización sobre las conflictivas que, habitualmente, se presentan adheridas a núcleos rígidos o uniformes de simbolización. Cuando las restricciones en los procesos de simbolización adquieren tal prevalencia y fijeza, tiende a reducirse la potencialidad de la imaginación (Castoriadis, 1993a), como actividad de invención de representaciones singulares sobre la experiencia subjetiva. 
Desde esta perspectiva, el trabajo clínico en el tratamiento psicopedagógico intenta favorecer que cada sujeto pueda articular, de un dúctil y autónomo, sus procesos singulares de invención (imaginativa) de sentidos subjetivos sobre la experiencia, con los recursos simbólicos necesarios que organizan su mediación en representaciones de palabra (reguladas por el proceso secundario) para su transmisibilidad y puesta en intercambio con los otros semejantes.

\section{La escritura narrativa en la clínica psicopedagógica}

Diferentes autores convergen en puntualizar que el trabajo narrativo de la escritura involucra un carácter configurante, activo, en los procesos de construcción identitaria (White, 1992; Ricoeur, 1995; Arfuch, 2002a, 2002b; Klein, 2008, 2010).

Entre ellos, la Dra. Silvia Schlemenson (2009) destaca que la actividad narrativa realza, en la producción escritural, formas distintivas de elaboración de sentido sobre la experiencia que aluden a los procesos imaginativos, y por ende - a un tipo particular de posicionamiento subjetivo por parte del narrador, quien potencialmente altera - en el campo del relato - las formas instauradas de interpretar la experiencia.

El trabajo narrativo de la escritura comprende una actividad creadora, en tanto articula aspectos de la subjetividad del narrador en el proceso mismo de construcción de la trama, a través de la cual interpreta - en forma singular - la heterogeneidad del acontecer temporal (White, 1992; Ricoeur, 1987).

Para Ricoeur (1995), el trabajo de configuración de la trama, implica ante todo- una actividad de mediación de la imaginación creadora, ya que identifica la perspectiva subjetiva del autor en el proceso de construcción de sentido.

Irene Klein (2007), por su parte, afirma que un sujeto que escribe inventa un yo que narra. En la misma línea, Emanuele Coccia (2008) considera que el trabajo de representación implica, ante todo, un acto de invención, de imaginación.

Esta perspectiva interroga, sin dudas, la tradición objetivista del positivismo, e incorpora la subjetividad del narrador (sus marcas, posicionamientos, mociones afectivas) como eje activo del proceso (identificante) de elaboración de sentido.

En este punto, la escritura narrativa entrelaza - de modo indisociable aspectos de la objetividad, con interpretaciones subjetivas que se concretan 
cuando se potencia la imaginación y el posicionamiento autónomo del narrador (Schlemenson, 2009).

Irene Klein (2008) considera, asimismo, que la invención de una trama narrativa implica un trabajo activo de mediación entre acontecimiento y sentido, operando así de forma intermediaria entre la heterogeneidad de la experiencia temporal y el trabajo de simbolización a través de su puesta en relato: "El hacer narrativo re-significa el mundo en su dimensión temporal" (Ricoeur, 1987, p. 158).

Partiendo de este marco teórico, se sostiene que la incorporación de la escritura en el tratamiento, intenta favorecer alternativas de elaboración sobre las conflictivas psíquicas preponderantes de cada joven que restringen su acceso a la autonomía de pensamiento, la imaginación y la interrogación crítica; como procesos necesarios para el acceso satisfactorio a todo proceso de aprendizaje (Schlemenson, 2009).

El trabajo con la escritura se articula, en el encuadre clínico del tratamiento, en tres momentos (no necesariamente sucesivos) de cada sesión grupal (Rego, 2006).

En un primer momento, los terapeutas intentan promover los primeros intercambios dialógicos y despliegues asociativos sobre las distintas problemáticas traídas al grupo por cada paciente (dificultades en la escuela, aspectos posicionales en relación a las figuras parentales, relación con los otros semejantes, historización de eventos significativos, entre otras).

Las problemáticas no siempre adquieren formas de presentación verbal en el discurso manifiesto, sino que - muchas veces - suelen expresarse a través de aspectos inhibitorios (silencios, respuestas no verbales o inaudibles), o bien de descarga (desorganización del discurso), que - en ambos casos - terminan limitando la función intermediaria del discurso entre las problemáticas internas de cada sujeto y la representación simbólica de sus sentidos subjetivos (Álvarez, 2010).

En un segundo momento, la intervención de los terapeutas se focaliza en la construcción y formulación de una consigna de trabajo (individual, grupal y/o colectiva), que tendrá la función de propulsar (a través de la producción gráfica o escrita en el cuaderno) alternativas de entrelazamiento, entre los elementos indiciarios de las problemáticas particulares que cada joven pudo haber desplegado en los intercambios iniciales del encuentro, y los procesos de simbolización para su tramitación singular en la producción simbólica. La escritura se concreta en el cuaderno que cada 
integrante dispone en una caja compartida que se conserva en el servicio asistencial. Se considera al cuaderno como un espacio - resguardado por el encuadre - para la proyección de aspectos subjetivos en representaciones compartidas (Schlemenson, 2009).

Como tercer momento, una vez que los pacientes finalizan sus escritos, se intentan ampliar - a través de la promoción del trabajo asociativo y la puesta en intercambio de las distintas producciones - las oportunidades diseminativas (Derrida, 1989, 2008) sobre las conflictivas esbozadas en la actividad escritural. Las características del encuadre grupal crean, en este sentido, un espacio adecuado de trabajo para el despliegue de nuevas oportunidades de simbolización y elaboración de sentido sobre las conflictivas singulares de cada sujeto (Álvarez \& Grunin, 2010).

Los resultados de la investigación, permitieron discernir que las propuestas ficcionales de escritura potenciaban, a lo largo del tratamiento, oportunidades proyectivas significativas para el despliegue de la subjetividad en: los atributos asignados a los personajes presentados, en la elección de las situaciones narradas (en general ligadas a problemáticas identitarias características, como la tramitación del cuerpo puberal, la relación con el semejante y/o la puesta en cuestión del discurso de los adultos), así como en sus modos particulares de resolución al interior de la trama narrativa (Grunin, 2013). Dichas propuestas (ficcionales) movilizan espacios alternativos de autoría en los que circulan oportunidades de revuelta (Kristeva, 2001, 2002) sobre los modos instituidos de representar(se) y proyectar(se) temporalmente.

Se pudo destacar que, a diferencia de la escritura autobiográfica, en estos casos el afecto adquiría una mayor plasticidad de circulación en el trabajo representativo de los sujetos. El posicionamiento subjetivo del narrador adquiría, así, en el encuadre clínico, otras formas posibles de expresión que aludían a la invención de tramas, desenlaces y/o perspectivas alternativas sobre lo narrado. La ficción "distancia" al narrador de sí mismo y lo representa (potencialmente) en otros espacios, tiempos y/o personajes posibles con los que podrá identificarse para ensayar alternativas en las formas de representar(se). La ductilidad de los ensayos comprometidos en los escritos de los jóvenes consultantes, pudo definirse - por ejemplo - en los cambios introducidos en los posicionamientos de los personajes narrativos presentados, la proyección de adjetivaciones, atributos imaginativos y/o cualidades afectivas en la trama narrativa, el despliegue de nuevos conflictos potenciales, así como la apertura imaginativa de perspectivas y/o resoluciones alternativas sobre los mismos (Grunin, 2013). 
Entre las conflictivas tramitadas en la producción escritural, se destacaron núcleos temáticos significativos que circulaban por: la representación de los cambios puberales, la elaboración de la modalidad posicional vinculada a los enunciados parentales, la historización de experiencias significativas, la nominación de afectos en relación a las mismas, o bien la incorporación de nuevos emblemas identitarios, entre otros.

En síntesis, las propuestas escriturales de carácter ficcional (o bien referidas a ejes de terceridad) promovían - aunque no de forma unidireccional oportunidades novedosas de ensayo a través de la inscripción de una distancia óptima (entre el narrador y lo narrado), que permitía la integración de aspectos subjetivos, cualidades afectivas y atributos imaginativos en la organización narrativa de la historia (Grunin, 2013).

\section{La escritura de los márgenes en la clínica psicopedagógica}

Si bien, en los inicios de la investigación, el recorte de objeto se hallaba circunscripto a la exploración de la actividad narrativa y sus alcances en la tramitación de aspectos identitarios, prontamente el proceso mismo de análisis de la escritura de los pacientes jóvenes, permitió reconocer otras formas distintivas (y cualitativamente novedosas) de elaboración de sentidos identitarios y tramitación de sus conflictivas asociadas.

Se descubrió, entonces, que los púberes y adolescentes escribían (espontáneamente) en los márgenes de sus cuadernos de trabajo, en distintos momentos de las sesiones grupales de su tratamiento.

De este modo, durante el trayecto investigativo se distinguió que, no sólo la escritura narrativa comprometía procesos activos de ensayo (configuración y re-configuración) de atributos, referencias y posicionamientos en relación a las problemáticas identitarias de cada joven, sino que - también - la escritura que desplegaban en los márgenes de sus cuadernos (laterales, superiores, inferiores, tapas y contratapas) involucraba procesos cualitativamente diferenciales de simbolización y producción - en este caso figurativa - de marcas identitarias (Schlemenson \& Grunin, 2013).

La apropiación del margen, por parte de los jóvenes, adquiría especificidades cualitativamente diferenciales respecto al trabajo escrito que los mismos realizaban al interior del espacio central ("tradicional") del cuaderno. El trabajo escrito en los márgenes expresaba formas novedosas de investimiento de la actividad imaginativa que inauguraban modalidades singulares de producción de sentido, las cuales (se debe subrayar) no hallaban su origen 
como respuesta a la consigna de trabajo que el terapeuta proponía en cada sesión grupal, sino que adquiría un carácter espontáneo, y potencialmente creativo, en diversos momentos de la sesión, hallándose siempre el cuaderno a disposición de los pacientes.

El presente hallazgo investigativo permitió abrir nuevas vías de abordaje sobre los procesos heterogéneos de simbolización implicados en la producción de los jóvenes durante su tratamiento. El análisis del trabajo de simbolización involucrado en los márgenes del cuaderno de los jóvenes, permitió crear mediaciones conceptuales alternativas para comprender sus formas específicas de despliegue y/o restricción.

Por ejemplo, si bien este tipo de escritura se apuntala en la organización del código socialmente compartido, al mismo tiempo incorpora modos plásticos de apropiación del código, que se caracterizan por la combinación de recursos mixtos de figurabilidad (discursiva, gráfica, escritural), investidos al servicio del ensayo de nuevos códigos, emblemas y/o marcas de autoría de alcance identitario, características de la adolescencia.

Los alcances identitarios de esta forma particular de producción simbólica de los márgenes, presentaban características formales novedosas, que podían ser asociadas al tipo de escritura que los jóvenes concretan en el uso contemporáneo de las nuevas tecnologías de información y comunicación (celulares, twitter, facebook, entre otras redes sociales). Por ejemplo, los púberes y adolescentes consultantes solían combinar - en los márgenes de sus cuadernos - mixturas de condensación figural entre el lenguaje oral y escritural ("2MIL08”, "AMI.X.100. PRE"), prolongaban y/o reproducian signos escritos, abreviaban palabras, reemplazaban letras, alteraban las mismas, o bien las invertían (algunas con caracteres espejados), introducían ondulaciones, rellenos y relieves, entre tantos otros indicios convergentes.

La escritura de los márgenes involucraba, así, oportunidades inéditas de re-creación de marcas identitarias. Por ejemplo: ensayos de firmas (indicio paradigmática de esta forma de escritura), invención de emblemas identificatorios, re-creación del nombre propio y/o de pares, frases de canciones, escritura de apodos, figuras gráficas representativas de los cambios puberales, entre tantas otras.

El espacio central del cuaderno, que anuda cierta expectativa de organización y transmisibilidad social hacia los otros a través de la actividad narrativa, resultó - de este modo - colocado en tensión con el espacio (figurativo) de los márgenes, en tanto espacio proyectivo de la subjetividad donde se figuran procesos transicionales de elaboración de la conflictiva identificatoria y pulsional. 
Escribir en los márgenes propulsa procesos de investimiento de nuevas trayectorias y proyectos identificatorios. En este sentido, supone un escenario potencial de confrontación (Winnicott, 1979) entre lo instituido y lo instituyente, entre lo ya conocido y la creación de nuevos objetos de interés libidinal (muchos de ellos valorados socialmente). Según Julia Kristeva (2002), es en los "márgenes" donde se produce el advenimiento de lo desconocido, como aquello que adviene en tanto ruptura de lo instituido, como espacio de revuelta y potencialidad instituyente.

Se considera, en esta línea, que el espacio del margen (como espacio de trabajo identificatorio) se ofrece al "escritor" como un espacio alternativo para inventar, ensayar, formas diversas de representar(se) y vincularse con los centros establecidos, que promueven (potencialmente) nuevas oportunidades de simbolización.

Desde una perspectiva metapsicológica de los procesos de simbolización (Green, 1996), puede considerarse - entonces - que las condiciones para la complejización de la producción simbólica de los púberes y adolescentes, refieren a la posibilidad de incorporación de una mayor heterogeneidad y ductilidad psíquica para la integración del afecto en su actividad representativa, de construcción de sentido.

En conclusión, la heterogeneidad de la actividad representativa se enriquece al poder conjugar los procesos imaginativos de producción de sentido sobre la experiencia subjetiva, con la expectativa de investimiento de las herramientas simbólicas que ofrece la cultura para su organización y puesta en intercambio con los otros semejantes en mundo exterior.

\section{Aportes y conclusiones}

Los púberes y adolescentes con problemas de aprendizaje presentan modalidades restrictivas de simbolización que empobrecen sus modalidades de productividad simbólica, entre ellas la producción escritural.

a) Cuando prevalecen formas rigidas y uniformes de simbolización, la producción simbólica de los jóvenes consultantes expresa modalidades estereotipadas - o bien fragmentarias - de construcción de sentido, que empobrecen sus procesos de aprendizaje e interacción con los objetos sociales y de conocimiento.

b) El trabajo clínico del tratamiento psicopedagógico intenta promover transformaciones en sus modalidades rígidas de simbolización, que 
pueden evaluarse por la incorporación de una mayor ductilidad psíquica en sus procesos de simbolización y construcción de sentido sobre la experiencia subjetiva.

c) La incorporación de una mayor ductilidad psíquica en sus procesos de elaboración de sentido, genera oportunidades más dúctiles de conectividad entre sus modalidades heterogéneas de simbolización, que enriquecen sus producciones simbólicas.

d) Dichas transformaciones pueden caracterizarse longitudinalmente a lo largo del tratamiento - en las modalidades de expresión de su actividad representativa.

e) El análisis de la actividad escritural de los jóvenes consultantes, permitió conocer sus modalidades distintivas de simbolización, caracterizar sus restricciones posibles y dar cuenta de sus condiciones de complejización en la clínica.

f) La generación de alternativas dúctiles de simbolización, amplía las oportunidades proyectivas de la subjetividad para la tramitación de sus conflictivas identitarias.

g) El abordaje investigativo de la escritura adolescente permitió pesquisar dos maneras heterogéneas de productividad simbólica que referían a elementos distintivos del proceso identificatorio, expresados tanto en sus aspectos narrativos (del cuerpo central del cuaderno), como figurales (de sus márgenes, tapas, contratapas, bordes y pie de páginas).

h) La incorporación de la escritura narrativa en el tratamiento psicopedagógico con los jóvenes, genera alternativas de simbolización sobre las conflictivas psíquicas preponderantes que restringen la ductilidad de su acceso a los procesos imaginativos, reflexivos y de autonomía de pensamiento.

i) El hallazgo de la escritura en los márgenes permitió distinguir modalidades específicas de simbolización, y alternativas con respecto al tipo de escritura tradicional, que derivaban en la constitución de un espacio novedoso de autoría para la tramitación de los elementos indiciarios de sus problemáticas identitarias.

j) Cuando se amalgaman, la ficción en su actividad narrativa, y la 
figurabilidad en su actividad escritural (espontánea) de los márgenes, se potencian formas novedosas de su imaginación creadora, que habilitan el investimiento de la escritura como un espacio inédito de autoría, subjetivación y ensayo sobre sus procesos de construcción identitaria en plena transformación.

k) Los desarrollos de la investigación aportan, en síntesis, herramientas conceptuales transferibles al área clínica y educativa, destinadas a enriquecer sus formas de intervención sobre las problemáticas de simbolización de los jóvenes con problemas de aprendizaje.

\section{Referencias}

Álvarez, P. (2010). Los trabajos psíquicos del discurso. Buenos Aires: Teseo.

Álvarez, P., \& Grunin, J. (2010). Función encuadrante y problemáticas actuales de simbolización. Revista Universitaria de Psicoanálisis, Facultad de Psicología. Universidad de Buenos Aires, 10, 15-33.

Arfuch, L. (2002a). El espacio biográfico: dilemas de la subjetividad contemporánea. Buenos Aires: Fondo de Cultura Económica.

Arfuch, L. (Comp.). (2002b). Identidades, sujetos y subjetividades. Buenos Aires: Prometeo Libros.

Aulagnier, P. (1977). La violencia de la interpretación: del pictograma al enunciado. Buenos Aires: Amorrortu. (Publicación original en 1975.)

Aulagnier, P. (1984). El aprendiz de historiador y el maestro brujo: del discurso identificante al discurso delirante. Buenos Aires: Amorrortu.

Aulagnier, P. (1986). Un intérprete en busca de sentido. México: Siglo XXI.

Aulagnier, P. (1991). Construir(se) un pasado. Revista de Psicoanálisis (APdeBA), 13 (3), 441-497.

Aulagnier, P. (1994). Los destinos del placer. Buenos Aires: Paidós.

Cantú, G. (2011). Lectura y subjetividad en la clínica psicopedagógica. Buenos Aires: Noveduc. 
Castoriadis, C. (1993a). Lógica, imaginación, reflexión. In C. Castoriadis. El inconciente y la ciencia. (pp.21-50). Buenos Aires: Amorrortu.

Castoriadis, C. (1993b). Psicoanálisis y política. In C. Castoriadis. El mundo fragmentado. (pp. 91-102). Montevideo: Altamira.

Cayssials, A. (2010). ¿Cuali y/o Cuanti? Aportes para elaborar informes integrativos en psicología. Buenos Aires: Paidós.

Coccia, E. (2008). Filosofía de la imaginación: averroes y el averroísmo. Buenos Aires: Adriana Hidalgo.

Derrida, J. (1989). La escritura y la diferencia. Barcelona: Anthropos.

Derrida, J. (2008). Márgenes de la filosofía. Madrid: Cátedra.

Franco, Y. (2004). Castoriadis: imaginación radical y complejidad. In Hornstein, L. Proyecto terapéutico: de Piera Aulagnier al psicoanálisis actual. (pp.307-317). Buenos Aires: Paidós.

Frigerio, G. (2008). La división de las infancias: ensayo sobre la enigmática pulsión antiarcóntica. Buenos Aires: Del Estante.

González Rey, F. (1999). La investigación cualitativa en psicología: rumbos y desafíos. São Paulo: EDUC Editora.

González Rey, F. (2006). Investigación cualitativa y subjetividad. Guatemala: Oficina de Derechos Humanos del Arzobispado de Guatemala.

Green, A. (1994). De locuras privadas. Buenos Aires: Amorrortu.

Green, A. (1996). La metapsicología revisitada. Buenos Aires: Eudeba.

Green, A. (2000). Una teoría general de la representación. In A. Fine, \& J. Schaeffer (Ed.). Interrogaciones psicosomáticas. (pp. 48-63). Buenos Aires: Amorrortu.

Green, A. (2001). La nueva clínica psicoanalítica y la teoría de Freud: aspectos fundamentales de la locura privada. Buenos Aires: Amorrortu.

Green, A. (2010). El pensamiento clinico. Buenos Aires: Amorrortu.

Grunin, J. (2009). Procesos de simbolización y trabajo de historización en la adolescencia. Revista Cadernos de Psicopedagogia, 7 (12). 
Grunin, J. (2013). Escritura y proceso identificatorio en la clínica de púberes $y$ adolescentes con problemas de aprendizaje. Tesis doctoral, Universidad de Buenos Aires (UBA), Buenos Aires, Argentina.

Grunin, J., \& Schlemenson, S. (2011a). Alcances identificatorios de la escritura adolescente. Revista Psicología, Conocimiento y Sociedad, Facultad de Psicología de la Universidad de la República, Uruguay, 1 (3), 6-29.

Grunin, J., \& Schlemenson, S. (2011b). Trabajo identificatorio en la adolescencia: escritura narrativa y de los márgenes. Perspectivas en Psicología, Facultad de Psicología. Universidad Nacional de Mar del Plata, 8, 86-94.

Gutton, P. (1991). Adolescente. Reportaje por Fernando Urribarri. Revista Zona erógena, 6, 22-24.

Gutton, P. (1993). Lo puberal. Buenos Aires: Paidós.

Klein, I. (2007). La narración. Buenos Aires: Eudeba.

Klein, I. (2008). La ficción de la memoria: la narración de historias de vida. Buenos Aires: Prometeo.

Klein, I. (2010). El relato de vida. In G. Pampillo (Comp.) Escribir: antes yo no sabia que sabia. (pp. 117-158). Buenos Aires: Prometeo.

Kristeva, J. (1995). Las nuevas enfermedades del alma. Madrid: Cátedra.

Kristeva, J. (2001). La revuelta intima; literatura y psicoanálisis. Buenos Aires: Eudeba. (Publicación original en 1997.)

Kristeva, J. (2002). La locura, la revuelta y la extranjería. Entrevista con Julia Kristeva (por Armen Avanecían y Lucas Degryae). Revista Signos filosóficos, México, 4 (7), 279-294. (Publicación original en Le Philosophoire, 14, París, Le lisible et I'illisible, 2001).

Lewkowicz, I. (1999). Historización en la adolescencia. Cuadernos de APdeBA, Departamento de Niñez y Adolescencia, 1, 109-126. (Ponencia del 20 de agosto de 1997.)

Mijolla-Mellor, S. (1991). La escritura en secreto. Revista de psicoanálisis con niños y adolescentes, Buenos Aires, 1 (1), 81-91.

Morin, E. (2000). Introducción al pensamiento complejo. Barcelona: Gedisa. 
Palazzini, L. (2006). Movilidad, encierros, enrancias: avatares del devenir adolescente. In M. C. Rother Hornstein (Comp.). (2006). Adolescencias: trayectorias turbulentas. (pp.137-160). Buenos Aires: Paidós.

Rego, V. (2006). Tres momentos de un encuentro: reflexiones acerca del encuadre clínico en el tratamiento psicopedagógico grupal. In L. Wettengel \& G. Prol (Comp.). Tratamiento de los problemas en el aprendizaje. (pp. 2738). Buenos Aires: Noveduc.

Ricoeur, P. (1987). Tiempo y narración I: configuración del tiempo en el relato histórico. Madrid: Cristianidad.

Ricoeur, P. (1995). Tiempo y narración II: configuración del tiempo en el relato de ficción. México: Siglo XXI.

Rother Hornstein, M. C. (Comp.). (2006). Adolescencias: trayectorias turbulentas. Buenos Aires: Paidós.

Schlemenson, S. (2009). La clínica en el tratamiento psicopedagógico. Buenos Aires: Paidós.

Schlemenson, S., \& Grunin, J. (2013). Psicopedagogía clinica: propuestas para un modelo teórico e investigativo. Buenos Aires: Eudeba.

Sternbach, S. (2006). Adolescencias: tiempo y cuerpo en la cultura actual. In M. C. Rother Hornstein (Comp.). (2006). Adolescencias: trayectorias turbulentas. (pp. 51-79). Buenos Aires: Paidós.

Taylor, S. J., \& Bogdan, R. (1992). Introducción a los métodos cualitativos de investigación. Barcelona: Paidós.

Wald, A. (2010). Los procesos imaginativos en niños y niñas con problemas de aprendizaje. Psicología em Revista, 16 (3), 437-447.

White, H. (1992). El contenido de la forma: narratividad, discurso y representación histórica. Madrid: Paidós. (Publicación original en 1987.)

Winnicott, D. (1979). Realidad y juego. España: Gedisa. 